\title{
Lumen apposing metal stents are superior to plastic stents in pancreatic walled-off necrosis: a large international multicenter study
}

다 (우우

\begin{abstract}
Authors
Yen-I Chen ${ }^{1,15}$, Juliana Yang ${ }^{1}$, Shai Friedland², Ian Holmes², Ryan Law ${ }^{3}$, Amy Hosmer $^{3}$, Tyler Stevens ${ }^{4}$, Matheus C. Franco $^{4}$, Sunguk Jang ${ }^{4}$, Rishi Pawa ${ }^{5}$, Nihar Mathur ${ }^{5}$, Divyesh V. Sejpal ${ }^{6}$, Sumant Inamdar ${ }^{6}$, Arvind J. Trindade ${ }^{6}$, Jose Nieto $^{7}$, Tyler M. Berzin ${ }^{8}$, Mandeep Sawhney ${ }^{8}$, Michael L. DeSimone ${ }^{8}$, Christopher DiMaio ${ }^{9}$, Nikhil A. Kumta ${ }^{9}$, Sanchit Gupta $^{9}$, Patrick Yachimski ${ }^{10}$, Andrea Anderloni ${ }^{11}$, Todd H. Baron ${ }^{12}$, Theodore W. James ${ }^{12}$, Laith H. Jamil ${ }^{13}$, Mel A. Ona ${ }^{13}$, Simon K. Lo ${ }^{13}$, Srinivas Gaddam ${ }^{13}$, Markus Dollhopf ${ }^{14}$, Majidah A. Bukhari ${ }^{1}$, Robert Moran ${ }^{1}$, Olaya Brewer Gutierrez $^{1}$, Omid Sanaei ${ }^{1}$, Lea Fayad ${ }^{1}$, Saowanee Ngamruengphong ${ }^{1}$, Vivek Kumbhari ${ }^{1}$, Vikesh Singh ${ }^{1}$, Alessandro Repici $^{12}$, Mouen A. Khashab ${ }^{1}$
\end{abstract}

Institutions

1 Division of Gastroenterology and Hepatology, Johns Hopkins Medical Institutions, Baltimore, MD, USA

2 Division of Gastroenterology, Stanford University School of Medicine, Stanford, CA, USA

3 Division of Gastroenterology, University of Michigan, Ann Arbor, MI, USA

4 Department of Gastroenterology and Hepatology, Cleveland Clinic, Cleveland, $\mathrm{OH}$, USA

5 Division of Gastroenterology and Hepatology, Wake Forest School of Medicine, Winston Salem, NC, USA

6 Division of Gastroenterology, Hofstra Northwell School of Medicine, Northwell Health System, Manhasset, NY, USA

7 Division of Gastroenterology and Hepatology, Borland Groover Clinic, Jacksonville, FL, USA

8 Center for Advanced Endoscopy, Beth Israel Deaconess Medical Center and Harvard Medical School, Boston, MA, USA

9 Division of Gastroenterology and Hepatology, Mount Sinai, New York, NY, USA

10 Division of Gastroenterology, Hepatology and Nutrition, Vanderbilt University, Nashville, TN, USA

11 Digestive Endoscopy Unit, Humanitas Research Hospital, Rozzano, Milan, Italy

12 Division of Gastroenterology and Hepatology, University of North Carolina, Chapel Hill, NC, USA

13 Division of Digestive and Liver Diseases, Cedars Sinai Medical Center, Los Angeles, CA, USA

14 Division of Gastroenterology and Hepatology, Klinikum Neuperlach, Munich, Germany

15 Division of Gastroenterology and Hepatology, McGill University Health Center, McGill University, Montreal, QC, Canada

submitted 5.3.2018

accepted after revision 31.7.2018
Bibliography

DOI https://doi.org/10.1055/a-0828-7630 |

Endoscopy International Open 2019; 07: E347-E354

(c) Georg Thieme Verlag KG Stuttgart · New York

ISSN 2364-3722

Corresponding author

Mouen A. Khashab, MD, Johns Hopkins Hospital, 1800

Orleans Street, Sheikh Zayed Tower, Baltimore, MD 21287, USA

Fax: +1-514-934-8547

mkhasha1@jhmi.edu

\section{ABSTRACT}

Background and study aims The use of lumen apposing metal stents (LAMS) during EUS-guided transmural drainage (EUS-TD) of pancreatic walled-off necrosis (WON) has gained popularity. Data supporting their use in WON over plastic stents (PS), however, remain scarce. The aim of this study was to compare the clinical efficacy of LAMS (Axios, Boston Scientific) with PS in WON.

Patients and methods This was a multicenter, retrospective study involving 14 centers. Consecutive patients who underwent EUS-TD of WON (2012-2016) were included. The primary end point was clinical success defined as WON size $\leq 3 \mathrm{~cm}$ within a 6 -month period without need for percutaneous drainage (PCD) or surgery.

Results A total of 189 patients (mean age 55.2 \pm 15.6 years, $34.9 \%$ female) were included (102 LAMS and 87 PS). Technical success rates were similar: $100 \%$ in LAMS and $98.9 \%$ in PS $(P=0.28)$. Clinical success was attained in $80.4 \%$ of LAMS and $57.5 \%$ of PS $(P=0.001)$. Rate of PCD was similar (13.7\% LAMS vs. $16.3 \% \mathrm{PS}, P=0.62)$, while PS was associated with a greater need for surgery (16.1\% PS vs. $5.6 \%$ LAMS, $P=0.02)$. Adverse events (AEs) were observed in $9.8 \%$ of LAMS and $10.3 \%$ of PS $(P=0.90)$ and 
were rated as severe in $2.0 \%$ and $6.9 \%$, respectively $(P=$ 0.93). After excluding patients with $<6$ months follow-up, the rate of WON recurrence following initial clinical success was greater with PS (22.9\% PS vs. $5.6 \%$ LAMS, $P=0.04)$.
Conclusions When compared to PS, LAMS in WON is associated with higher clinical success, shorter procedure time, lower need for surgery, and lower rate of recurrence.

\section{Introduction}

Acute necrotizing pancreatitis occurs in up to $20-40 \%$ of acute pancreatitis [1,2]. Pancreatic parenchymal or extraparenchymal necrosis can lead to tissue liquefaction and subsequent organization with a thin rim of granulation tissue otherwise known as pancreatic walled-off necrosis (WON). WON can be associated with substantial morbidity and mortality, especially when infected [2-5]. Although generally asymptomatic, WON can result in abdominal pain, early satiety, gastric outlet and biliary obstruction, vascular occlusion, fistula formation, and the development of pseudoaneurysm and bleeding. Traditionally, WON has been managed surgically; however, surgical necrosectomy is associated with substantial morbidity and mortality, particularly when pursued early $[6,7]$. More recently, a step-up approach consisting of a minimally invasive modality, such as percutaneous (PCD) or endoscopic drainage has been shown to decrease morbidity and mortality, and is advocated over surgery as first-line treatment by consensus guidelines $[5,6]$. A peroral endoscopic approach has gained popularity with the advent of therapeutic EUS and the development of novel stents that allow for drainage and direct endoscopic necrosectomy (DEN) [8,9].

Generally, endoscopic drainage of WON is performed with plastic stents. These stents, however, are limited by their small diameter leading to increased risk of stent obstruction [1013]. While studies using a combination of plastic stents and PCD for the drainage of WON have shown high clinical success rates $[14,15]$, the same day integrated delivery of these interventions is not feasible for most centers. Recently, lumen-apposing metal stents (LAMS) have been introduced for the management of pancreatic fluid collections (PFC), including WON [16]. The main advantages of LAMS include their large diameters that allow for greater drainage and permit DEN, while the bi-flanged design prevents stent migration. LAMS placement for PFCs is associated with excellent outcomes; however, most studies have included both pseudocysts and WON, which have vastly different natural histories and response to management [17-21]. In addition, comparative data for LAMS vs. plastic stents (PS) specifically for treatment of WON are scarce.

There are currently three types of LAMS on the market, namely the Axios (Boston Scientific, Marlborough, MA, United States), Nagi, and Spaxus (Taewoong, Medical, Ilsan, South Korea) stents. The goal of this large international retrospective study was to compare the clinical outcome of endoscopically placed LAMS (Axios) with plastic stents in the management of WON. The Nagi and Spaxus stents were not included given their unavailability in the USA.

\section{Materials and methods}

This is an international, multicenter retrospective study involving 14 centers ( 12 in North America, 2 in Europe). Consecutive patients who underwent EUS-guided WON drainage with either LAMS or PS between January 2012 and August 2016 were included. A total of 17 patients included in this study who underwent insertion of LAMS for WON were reported in a previous publication [27]. WON was defined as per the Atlanta classification as an organized collection with both liquid and necrotic solid components (on CT, MRI, and/or EUS) developing approximately 4 weeks post-acute pancreatitis [22]. Patients with pancreatic pseudocysts, defined as an organized collection without solid components developing 4 weeks post-acute pancreatitis [22], and patients with less than 30 days follow-up were excluded. The institutional review board at each center approved this study.

Patients were identified using center-specific endoscopic or billing databases. Electronic chart reviews were performed to capture variables such as demographics, WON location, size, percentage of necrosis, extension into the paracolic gutter, presence of main pancreatic duct disruption or leak based on endoscopic retrograde cholangiopancreatography (ERCP) findings, etiology of pancreatitis, indication for drainage, stent type, size, and number, drainage approach (transgastric, transduodenal, transesophageal), technical success, procedure time, need for DEN, number of DEN sessions (if required), use of hydrogen peroxide irrigation, insertion of nasocystic tubes, need for percutaneous drainage (PCD), size of PCD, clinical success, successful stent removal following clinical success, ERCP performed within 30 days pre- or post-index procedure and insertion of transpapillary pancreatic stents, stent obstruction, stent migration with stent displacement confirmed via endoscopy, WON recurrence, need for surgery, adverse events with severity graded according to the ASGE lexicon [23], length of hospitalization, and total follow-up time in days.

\section{Drainage of walled-off pancreatic necrosis with the lumen apposing metal stent}

As per standard practice, written informed consent was obtained from all patients before the procedure. Prophylactic antibiotics were administered peri-procedurally per institutional protocol. A linear echoendoscope was then inserted to first assess the location, size, and amount of necrosis. Color Doppler was then used to assess surrounding vascular structures. The distance between the EUS-probe and WON was then ascertained and drainage performed only if it was $<1 \mathrm{~cm}$. LAMS insertion with cautery assistance into the WON cavity was followed by deployment of the distal flange then the proximal flange under 
EUS or endoscopic guidance. If a non-cautery assisted LAMS was used, then the WON was first punctured with a 19-gauge needle followed by coiling of 0.025 or 0.035 inch guidewire in the WON under fluoroscopic guidance, tract dilation, and insertion of the LAMS. At the discretion of the endoscopist, the stent could then be dilated to the desired size with a wire guided radial expansion balloon. Direct endoscopic necrosectomy could then be performed at the index procedure or more commonly during a subsequent procedure. DEN was performed through the LAMS with a forward viewing gastroscope. Necrotic tissue was then debrided using various accessories. Irrigation of the WON cavity with dilute hydrogen peroxide and insertion of a nasocystic tube was performed at the discretion of the endoscopist.

\section{Drainage of walled-off pancreatic necrosis with plastic stents}

Following the appropriate peri-procedural care as described above, the WON was assessed with a linear echoendoscope and the cavity punctured with a 19-gauge fine-needle aspiration (FNA) needle from the stomach or duodenum. A 0.025 0.035 inch guidewire was then inserted and coiled inside the WON cavity under fluoroscopic guidance. Tract dilation was then performed either with cystotome or radial expansion balloon to facilitate stent placement. Double pigtail plastic stents were inserted over the wire under fluoroscopic guidance. The number and size of stents used were at the discretion of the endoscopist. When required, DEN was performed once the cystogastrostomy/duodenostomy tract had matured. This was done by first removing the stents followed by dilation of the tract with a radial expansion balloon and insertion of a forward viewing gastroscope through the tract for DEN. Plastic stents were then placed after completion of the necrosectomy. The use of nasocystic tube drainage and/or hydrogen peroxide irrigation was at the discretion of the endoscopist.

\section{End points}

The primary end point was the rate of clinical success defined as symptom resolution and decrease in size of the WON to $\leq 3 \mathrm{~cm}$ in diameter within 6 months without the need for PCD or surgery. Secondary end points included rate of technical success, adverse events with severity graded per the ASGE Lexicon [23], stent dysfunction due to migration or occlusion, WON recurrence (defined as recurrent symptoms and increase in WON diameter $>3 \mathrm{~cm}$ ), and need for surgery defined as any surgical intervention (minimally invasive or open) for the management of unresolved WON or complications arising from endoscopic or percutaneous drainage.

\section{Statistical analysis}

Continuous variables were reported as mean \pm standard deviation (or median and range, where appropriate). Categorical variables were reported as proportions and $95 \% \mathrm{Cls}$, with inferential analysis performed using chi-squared testing. A level of significance of $P<0.05$ was adopted for all inferential testing. A total of 13 independent variables considered to be clinically relevant by the investigators in predicting clinic success were identified for standard stepwise logistic regression analysis. These included age, gender, WON location, WON size, percent necrosis, extension of WON into paracolic gutter, etiology of pancreatitis, stent type (LAMS vs. PS), drainage approach (transgastric vs. transduodenal), endoscopic necrosectomy performed, use of hydrogen peroxide irrigation, ERCP performed $<30$ days after drainage, and presence of pancreatic duct disruption. Variables with a $P$ value of less than 0.25 in the univariable analysis were carried forward into the multivariable analysis.

\section{Results}

A total of 189 patients (mean age $55 \pm 16$ years, $34.9 \%$ female) were identified with 102 cases of LAMS and 87 cases of PS ( $\triangleright$ Table 1 ). Etiologies of acute pancreatitis were $42.0 \%$ gallstone pancreatitis, $30.3 \%$ idiopathic, $22.3 \%$ alcohol induced, $3.7 \%$ post ERCP, $1.1 \%$ trauma, and $0.5 \%$ autoimmune ( $\triangleright$ Table 2). Primary indications for drainage were abdominal pain in $49.5 \%$, infected WON in $26.6 \%$, gastric outlet obstruction in $12.2 \%$, early satiety in $5.9 \%$, rapid increase in WON size in $3.7 \%$, biliary obstruction in $1.1 \%$, and other causes in $1.1 \%$. WON was located at the head in $17.1 \%$, the body in $66.3 \%$, the tail in $11.8 \%$, involving the entire pancreas in $4.3 \%$, and extrapancreatic in $0.5 \%$. The mean diameter was $120.9 \pm 51.4 \mathrm{~mm}$. Percent necrosis as estimated on CT scan or MRI (available in 149 patients) was $0-25 \%$ in 33 patients, $25-50 \%$ in 52 patients, $50-75 \%$ in 41 patients, and $>75 \%$ in 23 patients.

Paracolic gutter extension was noted in $30.7 \%$ LAMS and $39.5 \%$ PS $(P=0.21)$, while disconnected pancreatic duct, as di-

- Table 1 Distribution of data by center and stent type.

\begin{tabular}{|c|c|c|c|}
\hline Center & LAMS & PS & Total \\
\hline 1 & 20 & 6 & 26 \\
\hline 2 & 8 & 4 & 12 \\
\hline 3 & 8 & 18 & 26 \\
\hline 4 & 4 & 5 & 9 \\
\hline 5 & 9 & 10 & 19 \\
\hline 6 & 3 & 1 & 4 \\
\hline 7 & 26 & 0 & 26 \\
\hline 8 & 4 & 17 & 21 \\
\hline 9 & 1 & 5 & 6 \\
\hline 10 & 5 & 2 & 7 \\
\hline 11 & 2 & 8 & 10 \\
\hline 12 & 2 & 3 & 5 \\
\hline 13 & 2 & 0 & 2 \\
\hline 14 & 8 & 8 & 16 \\
\hline Total & 102 & 87 & 189 \\
\hline
\end{tabular}


- Table 2 Baseline demographic and walled-off pancreatic necrosis characteristics.

\begin{tabular}{|c|c|c|c|}
\hline & LAMS $(n=102)$ & PS $(n=87)$ & $P$ value \\
\hline Age, mean $\pm S D$, years & $54 \pm 16$ & $57 \pm 15$ & 0.213 \\
\hline Female gender, \% & 53.0 & 47.0 & 0.850 \\
\hline \multicolumn{3}{|l|}{ Etiology of pancreatitis, \% } & \multirow[t]{7}{*}{0.497} \\
\hline - Gallstone pancreatitis & 42.6 & 41.4 & \\
\hline - Idiopathic & 17.8 & 20.7 & \\
\hline - Alcohol induced & 26.7 & 17.2 & \\
\hline - Post ERCP & 3.0 & 4.6 & \\
\hline - Trauma & 1.0 & 1.1 & \\
\hline - Autoimmune & 1.0 & 0 & \\
\hline \multicolumn{3}{|l|}{ WON location, \% } & \multirow[t]{6}{*}{0.249} \\
\hline - Head & 18.8 & 15.1 & \\
\hline - Body & 66.3 & 66.3 & \\
\hline - Tail & 7.9 & 16.3 & \\
\hline - Entire pancreas & 5.9 & 2.3 & \\
\hline - Extrapancreatic & 1.0 & 0 & \\
\hline WON size, mm & $111.4 \pm 43.6$ & $135.0 \pm 58.1$ & 0.213 \\
\hline \multicolumn{3}{|l|}{ Percent necrosis, \% } & \multirow[t]{5}{*}{0.190} \\
\hline . $0-25 \%$ & 16.0 & 29.4 & \\
\hline . $25-50 \%$ & 40.7 & 27.9 & \\
\hline . $50-75 \%$ & 27.2 & 27.9 & \\
\hline - $75-100 \%$ & 16.0 & 14.7 & \\
\hline Paracolic gutter extension, \% & 30.7 & 39.5 & 0.206 \\
\hline Disconnected duct, \% & 15.8 & 31.4 & 0.114 \\
\hline
\end{tabular}

agnosed on ERCP performed in 73 patients, was seen in $15.8 \%$ LAMS and $31.4 \%$ PS $(P=0.11)$ ( $>$ Table 2$)$. Percent necrosis was also comparable between the two groups with $54.7 \%$ of LAMS and $45.3 \%$ of PS having $>50 \%$ necrosis.

\section{Procedure characteristics}

Transgastric stenting was the most common approach in both cohorts (95.1\% LAMS vs. 92.0\% PS, $P=0.462$ ) ( $\triangleright$ Table 3 ). Procedure time was significantly shorter with LAMS $(50.4 \pm 26.5$ minutes vs. $64.6 \pm 34.0$ minutes, $P=0.003)$. DEN was more frequently performed in the LAMS group (81.4\% LAMS vs. $48.4 \%$ PS, $P<0.001)$. In patients who underwent DEN, the mean numbers of sessions were $1.9 \pm 1.4$ in LAMS and 2.6 \pm 2.9 in PS $(P=$ 0.09 ). Hydrogen peroxide irrigation was undertaken in $51.0 \%$ and $4.6 \%$ of patients with LAMS and PS, respectively $(P<$ 0.001 ), while nasocystic drain insertion was comparable between the two groups ( $3.9 \%$ LAMS vs. $6.9 \%$ PS, $P=0.36$ ). The total number of endoscopic procedures before stent removal was similar between the two groups $(2.6 \pm 1.5$ LAMS vs. $3.1 \pm$
1.5 PS, $P=0.129)$. ERCP was performed in $34.3 \%$ LAMS and 41.4\% PS $(P=0.32)$, with main pancreatic duct stent insertion in $18.6 \%$ LAMS and $27.6 \%$ PS $(P=0.14)$. Pancreatic stent was deemed to have bridged the pancreatic duct leak in $56.3 \%$ and $36.4 \%$ in LAMS and PS, respectively $(P=0.22)$.

\section{Clinical end points and adverse events}

Technical success was achieved in $100.0 \%$ of procedures in the LAMS group compared to $98.9 \%$ of procedures in the PS group $(P=0.28)(\triangleright$ Table 4). Post-procedural mean length of stay was not significantly different between the two groups (10.4 \pm 27.1 days for LAMS vs. $10.2 \pm 16.4$ days for PS, $P=0.93$ ). Clinical success was attained in $80.4 \%$ of LAMS patients compared to $57.5 \%$ of PS patients $(P=0.001)$. The need for percutaneous drainage was similar between the two groups (13.7\% LAMS vs. $16.3 \% P=0.624)$, while the use of PS was associated with significantly greater need for surgery (16.1\% PS vs. 5.6\% LAMS, $P=$ 0.023). 
- Table 3 Procedure characteristics.

\begin{tabular}{|c|c|c|c|}
\hline & LAMS $(n=102)$ & PS $(n=87)$ & $P$ value \\
\hline \multicolumn{3}{|l|}{ Drainage approach, \% } & \multirow[t]{4}{*}{0.462} \\
\hline - Transgastric & 95.1 & 92.0 & \\
\hline - Transduodenal & 4.9 & 6.9 & \\
\hline - Transesophageal & 0 & 1.1 & \\
\hline Number of stents & $1.07 \pm 0.3$ & $2.3 \pm 0.8$ & $<0.001$ \\
\hline Total mean stent diameter, mm & 14.8 & 7.7 & $<0.001$ \\
\hline DEN performed, \% & 81.4 & 48.4 & $<0.001$ \\
\hline Mean number of DEN & $1.9 \pm 1.4$ & $2.6 \pm 2.9$ & 0.09 \\
\hline Hydrogen peroxide irrigation, \% & 51.0 & 4.6 & $<0.001$ \\
\hline Nasocystic drain, \% & 3.9 & 6.9 & 0.362 \\
\hline Total number of endoscopic procedures & $2.6 \pm 1.5$ & $3.1 \pm 1.5$ & 0.129 \\
\hline ERCP performed, \% & 34.3 & 41.4 & 0.317 \\
\hline Transpapillary pancreatic stent, \% & 18.6 & 27.6 & 0.143 \\
\hline Pancreatic stent bridges site of leak, \% & 56.3 & 36.4 & 0.224 \\
\hline
\end{tabular}

- Table4 Clinical outcomes.

\begin{tabular}{|l|c|c|c|}
\hline & LAMS (n=102) & PS (n=87) & P value \\
\hline Technical success, \% & 100.0 & 98.9 & 0.276 \\
\hline Clinical success, \% & 80.4 & 57.5 & 0.001 \\
\hline Post procedure length of stay, days & 10.4 & 10.2 & 0.930 \\
\hline Percutaneous drain, \% & 13.7 & 16.3 & 0.624 \\
\hline Surgical intervention, \% & 5.6 & 16.1 & 0.023 \\
\hline Recurrenc' 1 \% & 5.6 & 22.9 & 0.036 \\
\hline $\begin{array}{l}\text { LAMS, Lumen apposing metal stent; PS, plastic stent. } \\
\text { 1 After excluding patients with less than 6 months follow-up (total of 74 patients excluded: 52 LAMS and 22 PS). }\end{array}$ \\
\hline
\end{tabular}

A total of 19 adverse events were observed with an overall adverse event rate of $10.1 \%$. The rate of AEs was comparable between the two groups (9.8\% LAMS and $10.3 \%$ PS, $P=0.90$ ). Adverse events included bleeding $(n=10)$, peritonitis $(n=4)$, stent misdeployment $(n=1)$, perforation $(n=2)$, and other $(n=$ 2 ). In terms of severity, $26.3 \%$ were rated as mild, $31.6 \%$ moderate, and $42.1 \%$ severe. The rate of severe adverse events was $2.0 \%$ for LAMS and $6.9 \%$ for PS $(P=0.93)$. In terms of stent dysfunction, migration occurred in $2.9 \%$ LAMS and $6.9 \%$ PS ( $P=$ 0.20 ), while stent occlusion occurred in $20.6 \%$ LAMS vs. $12.6 \%$ PS $(P=0.15)$. In those who achieved clinical success, the use of PS was associated with higher rates of WON recurrence $(24.0 \%$ PS vs. $2.4 \%$ LAMS, $P<0.001)$. When excluding all patients with $<6$ months follow-up, recurrence rates remained higher in the PS cohort (22.9\% PS vs. 5.6\% LAMS, $P=0.04$ ) ( $\triangleright$ Table 4 ). Stent removal following clinical resolution was successful in all patients who were not lost to follow-up. The median length of fol- low-up was 144 days (IQR 87.8 - 200.25 days) for LAMS and 436 days (IQR 147 - 725 days) for PS.

\section{Predictors of clinical success}

Univariable analysis of the aforementioned variables identified the following factors as predictors of clinical success: size of the WON $(P=0.004)$, WON extension into the paracolic gutter $(P<0.001)$, stent type/LAMS vs. PS $(P=0.001)$, hydrogen peroxide irrigation $(P=0.02)$, and ERCP performed $<30$ days after WON drainage $(P=0.03)$. On multivariable analysis, the use of LAMS (OR 3.2, 95\%Cl: $1.3-8.0, P=0.01$ ) and the performance of ERCP $<30$ days after drainage (OR 3.4, 95\%Cl: $1.3-8.9, P=$ 0.01 ) were identified as independent positive predictors of clinical success while the presence of paracolic gutter WON extension (OR 0.3, 95\% Cl: $0.1-0.7, P=0.006$ ) was an independent negative predictor of clinical success ( $\triangleright$ Table 5). 
- Table 5 Multivariable analysis for predictors of clinical success.

\begin{tabular}{|l|l|l|}
\hline Variables & OR, 95\%CI & P value \\
\hline LAMS vs. PS & $3.2,1.3-8.0$ & 0.01 \\
\hline $\begin{array}{l}\text { ERCP performed < 30 days post } \\
\text { drainage }\end{array}$ & $3.4,1.3-8.9$ & 0.01 \\
\hline Paracolic gutter extension & $0.3,0.1-0.7$ & 0.006 \\
\hline WON size & $1.0,0.996-1.01$ & 0.3 \\
\hline Hydrogen peroxide irrigation & $2.2,0.7-6.6$ & 0.2 \\
\hline Age & $1.0,0.992-1.05$ & 0.2 \\
\hline $\begin{array}{l}\text { ERCP, endoscopic retrograde cholangiopancreatography; LAMS, Lumen ap- } \\
\text { posing metal stent; PS, plastic stent; WON, walled-off necrosis. }\end{array}$
\end{tabular}

\section{Discussion}

With the advent of LAMS and data supporting a minimally invasive step-up approach to WON over the surgical method [5, 8], endoscopic management of pancreatic fluid collections has gained popularity. In this international multicenter retrospective study, we demonstrate that the use of LAMS for treatment of WON is associated with higher clinical success $(80.4 \%$ vs. $57.5 \% P=0.001)$, shorter procedure time (50.4 minutes vs. 64.6 minutes), lower requirements for surgery (5.6\% vs. $16.1 \%)$, lower WON recurrence following clinical success (22.9\% vs. $5.6 \%$ ), and similar rates of AEs (9.8\% vs. $10.3 \%)$ when compared to PS.

The rates of clinical success in both LAMS (80.4\%) and PS (57.5\%) groups are in keeping with the current literature [11, $18,20,21,24-29]$. In the largest series of LAMS placement for WON, clinical success was achieved in $86.3 \%$ of 124 patients, while in a study involving 211 patients who underwent PS insertion, the clinical success rate was $63.2 \%$ [27, 29]. Unlike pancreatic pseudocysts, the main challenge in WON treatment is removal of solid necrotic components of the collection. Several advantages of LAMS include their large diameters and anti-migration properties. Interestingly, however, despite the larger stent diameter, stent occlusion was frequent in LAMS occurring in $20.6 \%$ of cases. The larger diameter likely allows a greater amount of solid debris to drain spontaneously; however, this also means that large solid components can build up within the stent leading to occlusion. Some experts have advocated the insertion of a double pigtail plastic stent through the LAMS or insertion of multiple LAMS (multigateway drainage) in order to minimize occlusion ( $\triangleright$ Fig. 1 ). Indeed, a small comparative retrospective study has suggested that the addition of a double pigtail plastic stent through the LAMS may be associated with fewer adverse events [30].

Another potential advantage of LAMS is its ability to facilitate additional modalities of treatment including direct endoscopic necrosectomy and hydrogen peroxide irrigation. In fact, the rates of both DEN and hydrogen peroxide irrigation were significantly greater in patients who underwent drainage with LAMS in our study. The performance of DEN has been shown to lead to higher rates of successful WON resolution than drainage alone and is associated with a lower need for administration of antibiotics, shorter length of hospital stay, and lower health care utilization [24, 31]. However, with the use of PS, DEN is difficult and cumbersome requiring stent removal and tract dilation leading to higher rates of AEs, up to $14-26 \%$ and even mortality in up to $7.5 \%$ of cases [24,32]. LAMS, on the other hand, allows for DEN to be performed through the stent without the risk associated with stent removal and tract dilation. In a large retrospective series of 78 patients undergoing DEN following LAMS insertion, the AE rate was $6.4 \%$ with three cases of stent migration, all of which were replaced without sequela, and two cases of bleeding controlled with interventional radiology embolization [27]. The large diameter of the LAMS also facilitates irrigation with dilute hydrogen peroxide, which may enhance breakdown of solid necrotic debris [33,34]. Although these adjuvant modalities probably play an important role in the management of WON, our multivariable analysis identified the use of LAMS as an independent predictor of clinical success while both DEN and hydrogen peroxide irrigation were not significant predictors.
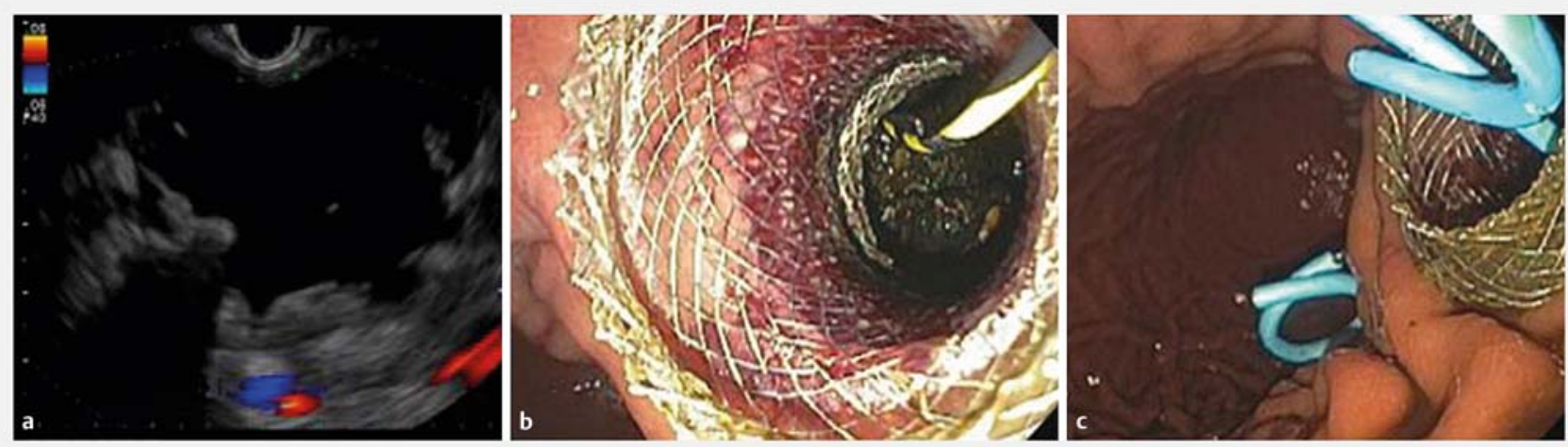

- Fig. 1 Multigateway drainage with insertion of two lumen apposing stents with additional plastic stents to prevent stent obstruction. a EUS image of a large pancreatic walled-off necrosis with extensive solid components. b Successful deployment of a lumen apposing stent forming a cystogastrostomy. c Two lumen apposing stents inserted with plastic double pigtail stents in situ. 
The performance of ERCP $<30$ days after WON drainage was a positive predictor of clinical success, while paracolic gutter WON extension was associated with a negative outcome thus highlighting the importance of a multi-modality and multi-disciplinary approach to WON [6]. The performance of ERCP following WON drainage may help identify patients with a disconnected pancreatic duct, which would then lead to either leaving the plastic double pigtail stents permanently or insertion of permanent double pigtail stents following LAMS removal. Interestingly, whether or not a transpapillary pancreatic stent was inserted during ERCP did not predict clinical outcome, which puts into question this practice while suggesting that ERCP is more important for proper assessment of integrity of the pancreatic duct than for pancreatic stent insertion.

The insertion of LAMS for WON drainage is also technically easier than PS. The availability of a cautery tip for LAMS insertion allows placement without the need for initial needle puncture, wire guidance, or tract dilation, all of which require device exchange. This may explain the significantly shorter procedural time observed in our study of $50.4 \pm 26.5$ minutes with LAMS vs. $64.6 \pm 34.0$ minutes with PS. A shorter procedure time was also noted in a previous report by Gornals et al. [35]. In addition, LAMS can be inserted without fluoroscopic guidance. Having fewer steps necessary for stent insertion may also lead to fewer adverse events. Our data, however, did not demonstrate a significant difference in AEs between the LAMS and PS group. There was a trend towards fewer severe AEs in the LAMS cohort, but this was not statistically significant. There has been a recent report of severe delayed bleeding with LAMS [36]. This is thought to be secondary to the rapid collapse of the collection and erosion of the wall against the flared flange of the LAMS. The authors of this report have therefore changed their protocol to shorten the interval between stent insertion and repeat axial imaging with immediate stent removal if the collection has collapsed. Overall, this appears to be a very rare complication of LAMS which was not observed in our study and other large case series [27,37]. Importantly, along with the higher clinical success rate, the use of LAMS was associated with a reduction in the need for surgery. The use of LAMS was also associated with fewer WON recurrences following initial clinical success. This may be due incomplete drainage of solid debris within the collection with PS leading to a subsequent recurrent inflammatory response and increase in collection size following stent removal. This observation is limited by the fact that patients from the PS cohort had longer durations of follow-up; however, this trend persisted even following exclusion of patients with $<6$ months follow-up.

The major limitation of this study is the inherent biases associated with its retrospective design. Also, only expert tertiary centers were involved limiting its generalizability to community practice. Many of the patients who underwent drainage of WON with PS came from an older cohort of patients and may not have benefited from improvements in the understanding and medical management of WON, including use of aggressive hydration for acute necrotizing pancreatitis, early enteral nutrition, judicious use of antibiotics, careful selection and timing of drainage, and the importance of DEN. In addition, follow-up in the PS cohort was significantly longer than in the LAMS group, which may have contributed to the higher WON recurrence and surgery rates seen in the PS group. However, even after excluding patients with less than 6 months follow-up from the LAMS cohort, our conclusion remained the same. Our data also suggest a shorter procedure time with LAMS; however, it is important to keep in mind that a standardized timekeeper was not available given the retrospective nature of this study and procedure time was defined as starting from insertion of the endoscope to removal of the endoscope and may not have accounted for additional procedures such as ERCP therefore limiting the robustness of this end point. Lastly, our results only apply to the Axios stent and cannot be generalized to other LAMS such as the Nagi and Spaxus stents. Strengths of our study include its multicenter design, large number of patients, and exclusion of patients with pseudocysts. It also provides comprehensive data on the different clinical and procedural factors that could influence outcomes including percent necrosis of the WON, presence of extension in the paracolic gutter, and whether or not concomitant ERCP was performed.

In conclusion, our data indicate that, when compared to PS, the use of LAMS in endoscopic drainage of WON is associated with higher clinical success, shorter procedure time, lower need for surgery, and lower rate of recurrence with a comparable rate of $\mathrm{AE}$. Prospective controlled trials are now needed to confirm our findings.

\section{Competing interests}

Yen-I Chen is a consultant for Boston Scientific; Shai Friedland is a consultant for Boston Scientific; Tyler Stevens is a consultant for Boston Scientific; Sunguk Jang is a consultant for Boston Scientific; Divyesh V. Sejpal is a consultant for Boston Scientific; Josee Nieto is a consultant for Boston Scientific; Tyler Berzin is a consultant for Boston Scientific; Christopher DiMaio is a consultant for Boston Scientific and for Medtronic; Nikhil Kumta is a consultant for Boston Scientific; Patrick Yachimski is a consultant for Boston Scientific; Todd H. Baron is a consultant and speaker for Boston Scientific and Olympus; Markus Dollhopf is a consultant for Boston Scientific; Vivek Kumbhari is a consultant for Boston Scientific; Vikesh Singh is a consultant for Abbvie, Ariel, and Akcea; Mouen A. Khashab is a consultant for Boston Scientific and Olympus; all other authors have no relevant conflicts of interest.

\section{References}

[1] Isayama H, Nakai Y, Rerknimitr R et al. Asian consensus statements on endoscopic management of walled-off necrosis Part 1: Epidemiology, diagnosis, and treatment. J Gastroenterol Hepatol 2016; 31 : 1546 1554

[2] Banks PA, Freeman ML. Practice Parameters Committee of the American College of Gastroenterology. Practice guidelines in acute pancreatitis. Am J Gastroenterol 2006; 101: 2379-2400 
[3] Tenner S, Baillie J, DeWitt J et al. American College of Gastroenterology guideline: management of acute pancreatitis. Am J Gastroenterol 2013; 108: 1400 - 1415; 1416

[4] Tyberg A, Karia K, Gabr M et al. Management of pancreatic fluid collections: A comprehensive review of the literature. World J Gastroenterol 2016; 22: $2256-2270$

[5] van Santvoort HC, Besselink MG, Bakker OJ et al. A step-up approach or open necrosectomy for necrotizing pancreatitis. NEJM 2010; 362: $1491-1502$

[6] Working Group IAP/APA Acute Pancreatitis Guidelines. IAP/APA evidence-based guidelines for the management of acute pancreatitis. Pancreatology 2013; 13: e 1-15

[7] Besselink MG, Verwer T], Schoenmaeckers E] et al. Timing of surgical intervention in necrotizing pancreatitis. Arch Surg 2007; 142: 1194 1201

[8] Bakker OJ, van Santvoort HC, van Brunschot S et al. Endoscopic transgastric vs surgical necrosectomy for infected necrotizing pancreatitis: a randomized trial. JAMA 2012; 307: 1053-1061

[9] Muthusamy VR, Chandrasekhara V. Committee ASoP. et al. The role of endoscopy in the diagnosis and treatment of inflammatory pancreatic fluid collections. Gastrointest Endosc 2016; 83: 481 - 488

[10] Bang JY, Wilcox CM, Trevino J et al. Factors impacting treatment outcomes in the endoscopic management of walled-off pancreatic necrosis. J Gastroenterol Hepatol 2013; 28: 1725-1732

[11] Ng PY, Rasmussen DN, Vilmann P et al. Endoscopic ultrasound-guided drainage of pancreatic pseudocysts: medium-term assessment of outcomes and complications. Endosc Ultrasound 2013; 2: 199-203

[12] Seewald S, Ang TL, Richter $\mathrm{H}$ et al. Long-term results after endoscopic drainage and necrosectomy of symptomatic pancreatic fluid collections. Dig Endosc 2012; 24: 36-41

[13] Varadarajulu S, Christein JD, Wilcox CM. Frequency of complications during EUS-guided drainage of pancreatic fluid collections in 148 consecutive patients. J Gastroenterol Hepatol 2011; 26: 1504-1508

[14] Gluck M, Ross A, Irani S et al. Dual modality drainage for symptomatic walled-off pancreatic necrosis reduces length of hospitalization, radiological procedures, and number of endoscopies compared to standard percutaneous drainage. J Gastrointest Surg 2012; 16: 248 256 ; discussion 256-257

[15] Ross AS, Irani S, Gan SI et al. Dual-modality drainage of infected and symptomatic walled-off pancreatic necrosis: long-term clinical outcomes. Gastrointest Endosc 2014; 79: 929-935

[16] Binmoeller KF, Shah J. A novel lumen-apposing stent for transluminal drainage of nonadherent extraintestinal fluid collections. Endoscopy 2011; 43: $337-442$

[17] Chandran S, Efthymiou M, Kaffes A et al. Management of pancreatic collections with a novel endoscopically placed fully covered self-expandable metal stent: a national experience (with videos). Gastrointest Endosc 2015; 81: 127-135

[18] Rinninella E, Kunda R, Dollhopf M et al. EUS-guided drainage of pancreatic fluid collections using a novel lumen-apposing metal stent on an electrocautery-enhanced delivery system: a large retrospective study (with video). Gastrointest Endosc 2015; 82: 1039-1046

[19] Shah RJ, Shah JN, Waxman I et al. Safety and efficacy of endoscopic ultrasound-guided drainage of pancreatic fluid collections with lumen-apposing covered self-expanding metal stents. Clin Gastroenterol Hepatol 2015; 13: 747-752

[20] Siddiqui AA, Adler DG, Nieto J et al. EUS-guided drainage of peripancreatic fluid collections and necrosis by using a novel lumen-apposing stent: a large retrospective, multicenter U.S. experience (with videos). Gastrointest Endosc 2016; 83: 699-707

[21] Walter D, Will U, Sanchez-Yague A et al. A novel lumen-apposing metal stent for endoscopic ultrasound-guided drainage of pancreatic fluid collections: a prospective cohort study. Endoscopy 2015; 47: $63-67$

[22] Banks PA, Bollen TL, Dervenis $C$ et al. Classification of acute pancreatitis - 2012: revision of the Atlanta classification and definitions by international consensus. Gut 2013; 62: 102-111

[23] Cotton PB, Eisen GM, Aabakken L et al. A lexicon for endoscopic adverse events: report of an ASGE workshop. Gastrointest Endosc 2010; 71: $446-454$

[24] Gardner TB, Chahal P, Papachristou Gl et al. A comparison of direct endoscopic necrosectomy with transmural endoscopic drainage for the treatment of walled-off pancreatic necrosis. Gastrointest Endosc 2009; 69: 1085 - 1094

[25] Lin H, Zhan XB, Sun SY et al. Stent selection for endoscopic ultrasound-guided drainage of pancreatic fluid collections: a multicenter study in China. Gastroenterol Res Pract 2014; 2014: 193562

[26] Rana SS, Bhasin DK, Rao C et al. Non-fluoroscopic endoscopic ultrasound-guided transmural drainage of symptomatic non-bulging walled-off pancreatic necrosis. Dig Endosc 2013; 25: 47-52

[27] Sharaiha RZ, Tyberg A, Khashab MA et al. Endoscopic therapy with lumen-apposing metal stents is safe and effective for patients with pancreatic walled-off necrosis. Clin Gastroenterol Hepatol 2016; 14: $1797-1803$

[28] Siddiqui AA, Kowalski TE, Loren DE et al. Fully covered self-expanding metal stents versus lumen-apposing fully covered self-expanding metal stent versus plastic stents for endoscopic drainage of pancreatic walled-off necrosis: clinical outcomes and success. Gastrointest Endosc 2017; 85: 758 - 765

[29] Varadarajulu S, Bang JY, Phadnis MA et al. Endoscopic transmural drainage of peripancreatic fluid collections: outcomes and predictors of treatment success in 211 consecutive patients. J Gastrointest Surg 2011; 15: $2080-2088$

[30] Puga M, Consiglieri CF, Busquets ] et al. Safety of lumen-apposing stent with or without coaxial plastic stent for endoscopic ultrasoundguided drainage of pancreatic fluid collections: a retrospective study. Endoscopy 2018; 50: $1022-1026$

[31] Kumar N, Conwell DL, Thompson CC. Direct endoscopic necrosectomy versus step-up approach for walled-off pancreatic necrosis: comparison of clinical outcome and health care utilization. Pancreas 2014; 43: 1334 - 1339

[32] Seifert H, Biermer M, Schmitt W et al. Transluminal endoscopic necrosectomy after acute pancreatitis: a multicentre study with longterm follow-up (the GEPARD Study). Gut 2009; 58: 1260-1266

[33] Abdelhafez M, Elnegouly M, Hasab Allah MS et al. Transluminal retroperitoneal endoscopic necrosectomy with the use of hydrogen peroxide and without external irrigation: a novel approach for the treatment of walled-off pancreatic necrosis. Surg Endosc 2013; 27: 3911 3920

[34] Siddiqui AA, Easler J, Strongin A et al. Hydrogen peroxide-assisted endoscopic necrosectomy for walled-off pancreatic necrosis: a dual center pilot experience. Dig Dis Sci 2014; 59: 687-690

[35] Gornals JB, De la Serna-Higuera C, Sanchez-Yague A et al. Endosonography-guided drainage of pancreatic fluid collections with a novel lumen-apposing stent. Surg Endosc 2013; 27: 1428-1434

[36] Bang JY, Hasan M, Navaneethan U et al. Lumen-apposing metal stents (LAMS) for pancreatic fluid collection (PFC) drainage: may not be business as usual. Gut 2017; 66: $2054-2056$

[37] Zeissig S, Sulk S, Brueckner S et al. Severe bleeding is a rare event in patients receiving lumen-apposing metal stents for the drainage of pancreatic fluid collections. Gut 2018: doi:10.1136/gutjnl-2018316581 [Epub ahead of print] pii: gutjnl-2018-316581 